Article - Human and Animal Health

\title{
Area Triangulation Method for Automatic Detection of Venous Emptying Maneuvers
}

\section{Ignacio Sánchez-Gendriz ${ }^{1, *}$}

https://orcid.org/0000-0002-3885-9510

\section{Hernández-Seoane ${ }^{2}$}

\section{A. Sóñora-Mengana ${ }^{2}$}

\section{A. Pascau-Simón ${ }^{2}$}

${ }^{1}$ Brain Institute, Federal University of Rio Grande do Norte, Natal, Brazil; ${ }^{2}$ Centro de Biofísica Médica, Universidad de Oriente, Santiago de Cuba, Cuba;

Received: 2018.09.28; Accepted: 2019.02.26.

\section{HIGHLIGHTS}

- Manual annotations on physiological signals are time consuming and prone to human bias.

- Two methods for automatic analysis of photoplethysmographic (PPG) waveforms are presented.

- Both methods comparable to humans, but Area Triangulation outperformed First Derivative method.

- Computer methods are effective to replace manual annotations on biomedical signals analysis.

Abstract: Venous refilling time (VRT) can diagnose the presence of venous diseases in lower limbs. In order to calculate VRT it is necessary to determine the End of the Emptying Maneuvers (EEM). First Derivative Method (FDM) can be employed for automatic detection of the EEM, but its sensitivity to artifacts and noise can degrade its performance. In contrast, studies report that Area Triangulation Method (ATM) evinces effectiveness in biosignals point finding. This work compares the exactness of ATM and FDM for recognition of the EEM. The annotations made by 3 trained human observers on 37 photoplethysmography records were used as a reference. Bland-Altman graphics supported the analysis of agreement among human observers and methods, which was complemented with Analysis of variance and Multiple Comparisons statistical tests. Results showed that ATM is more accurate than FDM for automatic detection of the EEM, with statistically significant differences ( $p$-value $<0.01)$.

Keywords: Area Triangulation Method; First Derivative Method; Photoplethysmographic; Venous Refilling Time. 


\section{INTRODUCTION}

Venous diseases (VDs) are among the sicknesses with the highest morbidity around the world, these disorders present considerable incidence both in female and male adults [1], [2]. There are studies that report not only a high prevalence of venous disorders, but also an increasing incidence [3]. The socioeconomics effects and impacts in patients' quality of live result considerable [2].

Muscle Pump Test (MPT) allows diagnosing VDs in early stages. In order to accomplish a MPT, the studied patient is seated on a chair. In this position, they should execute several continuous dorsiflexions (considered as the motion that reduces the angle between the foot and the leg). This exercise provokes lower limb venous emptying [4]. After blood emptying, a healthy venous system will be refilled only through capillaries. However, in the presence of sick veins part of the blood pumped by muscle contractions also refluxes through incompetent valves (as can be seen in Figure 1) and the veins refill more quickly [5]. Due to these facts, venous refilling time (VRT) helps discriminate between ailing and healthy veins. In a normal venous system, VRT generally lasts from 30 to 120 seconds, values lower than 20 seconds suggest a VD in lower limbs [5], [6].

Photoplethysmography (PPG), first introduced by Hertzman in 1937 [7], is a noninvasive and effective low-cost optical method that can be used to detect blood volume changes in the blood vessels [8]. Blood volume variation measured by PPG is based on the fact that near infrared light (in the range $800-1,000 \mathrm{~nm}$ ) irradiated on the human skin is significantly more absorbed by blood than by other tissues [9]. Consequently, the amount of infrared light reflected by the skin and registered by a photo-detector will be inversely related to the blood volume [9], [10]. In practical applications, PPG signal amplitude is often inverted and normalized (amplitude offset, followed by amplitude scaling). The amplitude reversal allows that blood volume changes induce directly proportional fluctuations on PPG waveform. On the other hand, amplitude normalization aims to minimize the influence of factors not directly related to blood volume changes. PPG is an indirect measurement, since the amount of reflected light also depends on factors such as thicknesses and skin pigmentation, local fat, among others [9], [11].

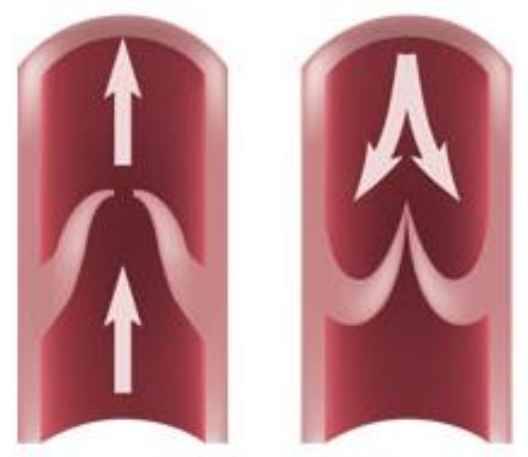

(a)

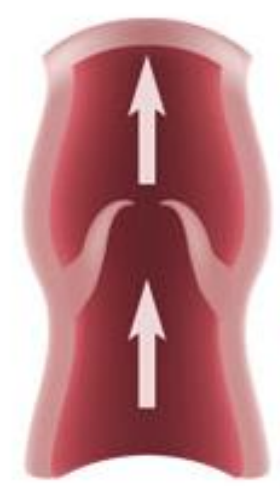

(b)

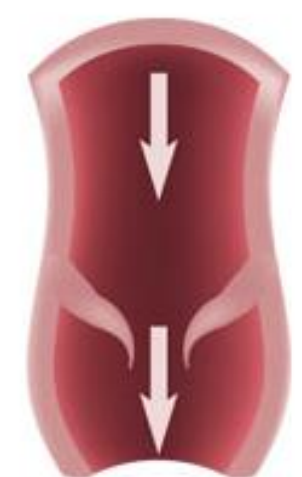

Figure 1 - Representation of lower limb venous valves functioning during calf muscle contraction and relaxation; (a) competent venous valve (prevents venous reflux); (b) deficient venous valve (abnormally dilated vein where incompetent valve fails to prevent venous reflux).

One of the clinical applications of PPG is for venous incompetence diagnosis [12]. Particularly, PPG can be employed for the measurement of VRT [13], [14], which is considered as reproducible approach for non-invasive assessment of lower limb venous function [6]. In order to calculate VRT on a venous PPG (VPPG) signal it is indispensable to determine the End of the Emptying Maneuvers (EEM), which can be defined as the instant determined by finalization of dorsiflexion executions during MPT, see Figure 2(b). 
Methods based on derivative have been traditionally used for critical point recognition on photoplethysmographic waveforms [15]. A previous study employed the First Derivative Method (FDM) for automatic detection of the EEM, results obtained showed a performance comparable with manual finding of the point [16]. Nevertheless, FDM is sensitive to noise and artifacts commonly contained in biomedical signals. On the other side, Area Triangulation Method (ATM) has been employed for point detection on arterial pulse waves signals [17]. In [17] the performance of ATM and a derivative based method were compared for automatic recognition of arterial pulse wave onsets, showing that ATM provides better results.

Based on cited facts, the present study proposes a new application for ATM: automatic detection of the EEM. Taking manuals annotations made by 3 trained human observers as reference, ATM and FDM are contrasted.

\section{MATERIAL AND METHODS}

\section{Experimental Protocol}

For the experiment, 37 VPPG records of 100 seconds duration were employed. Every record was sampled at $100 \mathrm{~Hz}$ and was acquired with Angiodin ${ }^{\circ}$ PD3000 photoplethysmograph during MPT. The implemented protocol consisted of the following steps:

1. Subject seated on a chair with their foot on the floor, leg slightly extended, the angle between the tibia and femur is approximately $110^{\circ}$, Figure 2(a).

2. PPG sensor placed $10 \mathrm{~cm}$ above the medial malleolus.

3. Wait for the stabilization of the VPPG signal, stage I Figure 2(b).

4. Execute 8 to 10 continuous dorsiflexions with 1s period between them stage II Figure 2(b).

5. Wait for venous refilling, in this region VPPG signal shows slow changes stage III Figure 2(b).

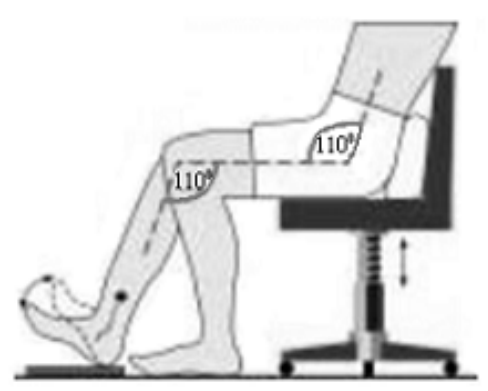

(a)

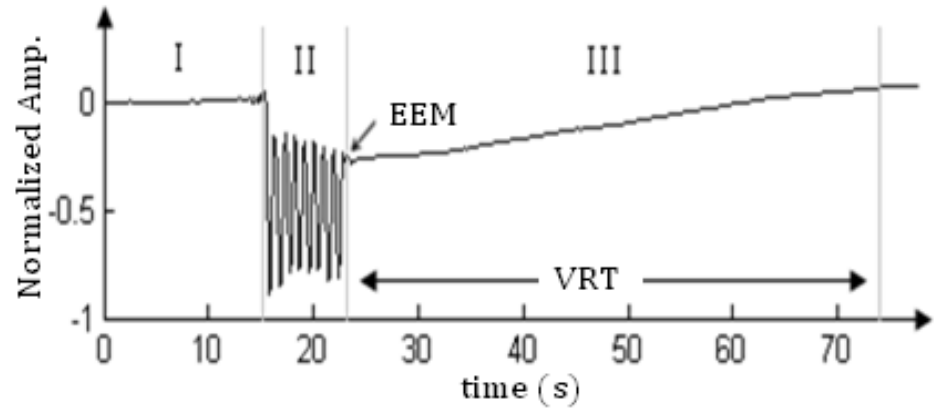

(b)

Figure 2 - (a) Subject and PPG sensor positioning during dorsiflexion executions in MPT; (b) VPPG signal from healthy person, acquired during MPT. Typical VPPG signals have three consecutives stages named: I - Basal state (waiting for of stabilization), II - Stage of dorsiflexions executions and III - Stage of venous refilling.

\section{Description of evaluated methods}

Both methods, FDM and ATM are based on similar facts, they include 3 steps:

1. Filtering and signal normalization

2. Determination of the local minimum induced by the last dorsiflexion (here on referred to as $\mathrm{T}_{\mathrm{min}}$ ).

3. Detection of the EEM from the point $T_{\min }$. This stage differentiates the two methods. 
First, the similarities between the two methods will be described and later the principal difference: the specific way of finding the EEM.

\section{Filtering and signal normalization}

Initially, VPPG signals are filtered in order to reduce noise and to eliminate unwanted frequency components. To accomplish this, a digital low pass FIR (Finite Impulse Response) filter was designed based on Parks-McClellan algorithm [18]. The filter is characterized by a cutoff frequency of $2 \mathrm{~Hz}$, a transition band of $0.3 \mathrm{~Hz}$ width, $0.1 \mathrm{~dB}$ passband ripple and a stopband attenuation of $-60 \mathrm{~dB}$.

For normalization of the VPPG signal two steps were applied:

1. Due to the fact that PPG represent an indirect measurement, the VPPG waveforms are proportionally converted to $0-1$ range (relative units), by applying an amplitude offset, followed by amplitude scaling.

2. Amplitude reversal (the inversion of the waveform amplitude).

After signal normalization, the amplitude of VPPG waveform ranges from 0 to -1 , representing relative units, which are denoted in the figures of the current work as normalized amplitude.

\section{Determination of $T_{\min }$}

Dorsiflexions provoke a progressive blood emptying of veins, and induces repeated local minima on the VPPG signal, see Figure 3(b). Both methods are based on the fact that the EEM appears shortly after $\mathrm{T}_{\min }$.

Every dorsiflexion is executed by two continuous movements, $m_{1}$ and $m_{2}$, Figure $3(a)$. One dorsiflexion lasts approximately $1 \mathrm{~s}$, so movements $m_{1}$ and $m_{2}$ are in the order of $1 / 2 \mathrm{~s}$ each. In order to determine if a local minimum is produced during a dorsiflexion execution, the first derivative $\mathrm{d}_{\mathrm{y}}(\mathrm{k})$ of every signal $\mathrm{y}(\mathrm{k})$ was calculated, as shown in equation (1). Then, from $\mathrm{d}_{\mathrm{y}}(\mathrm{k})$ a threshold value $\left(\mathrm{U}_{0}\right)$ was calculated as in equation (2).

Where $d_{y}^{-}(k)$ represents the negative values of $d_{y}(k)$ and $N$ is the number of $d_{y}^{-}(k)$ points. Later the local minima of the signal were determined. A local minimum detected in the VPPG signal was recognized as a minimum produced by a dorsiflexion execution if it fulfills the slope thresholding criterion, this criterion will be explained in more details below.

A linear least square fit was computed at $1 / 3 \mathrm{~s}$ segments for all the points before each local minimum detected on the VPPG signal, the fitted line was named $L_{0}$. The selection of the quantity of points needed to fit $L_{0}$ takes into account a representative length of the $m_{1}$ movement. Finally, $T_{\min }$ was located on the last minimum related to a dorsiflexion execution, where $L_{0}$ 's slope was less than or equal to $U_{0}$ (slope thresholding criterion). Figure $3(b)$ shows a $T_{\min }$ point located on a segment of a VPPG signal.

$$
\begin{gathered}
d_{y}(k)=y(k+1)-y(k), \\
U_{0}=\frac{\sum_{n=1}^{N} d_{y}^{-}(n)}{N},
\end{gathered}
$$




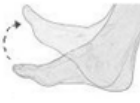

m1

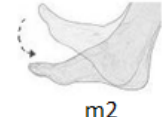

(a)

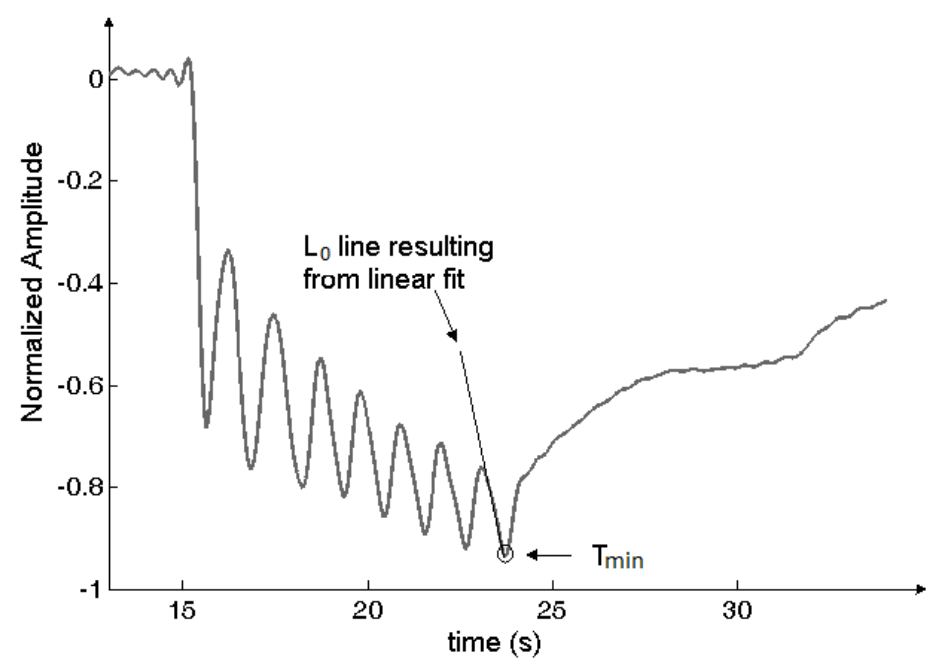

(b)

Figure 3 - (a) Movements that accomplish a complete dorsiflexion, (b) Oscillating decrease in VPPG signal originated by dorsiflexions and $T_{\min }$ point localization.

\section{Detection of the EEM by FDM}

The EEM was selected as the point on the VPPG signal that corresponds to the time of the first maximum of $d_{y}(k)$ after $T_{\min }$, as shown in Figure $4(a)$.

\section{Detection of the EEM by ATM}

ATM employs a recursive computation of a family of triangles with vertices $P 1, P 2$, and P3 located on VPPG signal. P1 and P3 are fixed points. P1 is located at $T_{\min }$ and P3 is the point located $1 \mathrm{~s}$ later, as shown in Figure $4(\mathrm{~b})$. The variable point P2 is displaced between $\mathrm{P} 1$ and $\mathrm{P} 3$. Then the EEM was selected as the value of P2 that forms the largest triangle area.

\section{Validating the methods}

Three trained human observers annotated the EEM on every VPPG signal five times each. The five annotations by the same observer on the same signal were not recorded consecutively. This was done to prevent the observers from annotating the EEM based on memory. The mean of the annotations for each signal by each observer was used as the final value for validating the methods. The Bland - Altman graphic method [19] was employed to assess inter-observer agreement and agreement among observers and methods.

Judging the inter-observer agreement is crucial. The human observers are taken as reference points for method evaluation, so the limits of agreement (LoA) [19] among observers should be narrow. Finally, the error of the methods was computed with respect to the observers. Based on Bland - Altman graphics and the computed errors, it was possible to determine the best method.

One-way and tow-way Analysis of variance (ANOVA) and multiple comparison tests [20] were used to evaluate significant statistical differences between observer annotations and between method's errors. One-way ANOVA, both in its parametric and non-parametric version (Kruskal-Wallis test) [21], were used to examine observer differences. In addition, one-way ANOVA was used for exploring differences between method's errors. Once detected that there are differences statistically significant between method's errors, subsequent multiple comparison test were then performed to determine which pairs of error groups are significantly different, and which are not. 


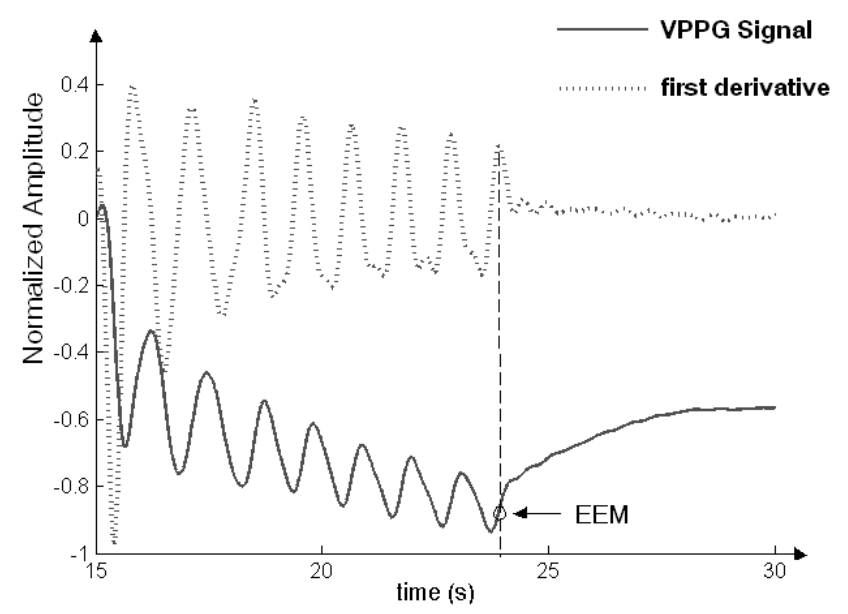

(a)

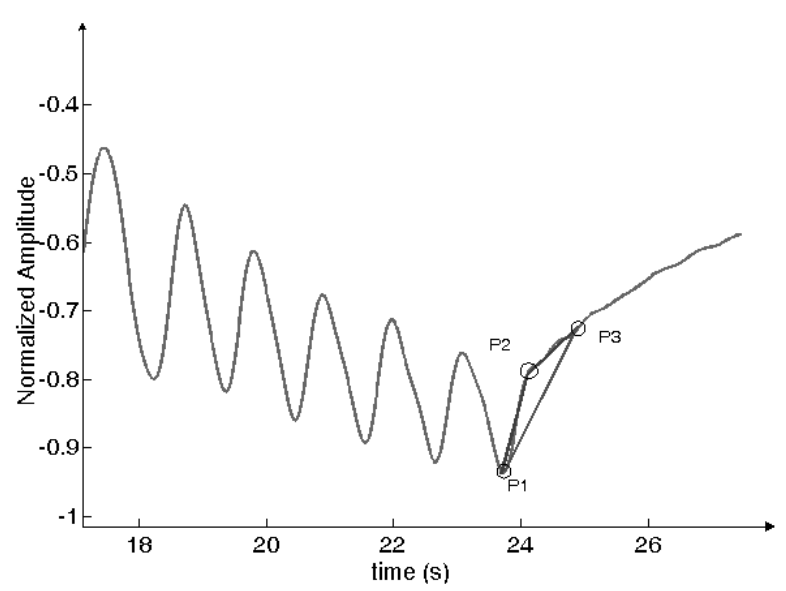

(b)

Figure 4. Representation of methods for automatic detection of EEM; (a) Detection by FDM, first derivative (enlarged) is superimposed on VPPG signal illustrating that EEM is selected as the instant for derivative peak fallowing $T_{\min }$; (b) Detection by ATM, EEM is selected as the value of P2 that forms the largest triangle area.

Matlab 7 was used for implementing both methods (FDM and ATM), and for statistical analysis performed in the present work.

\section{RESULTS AND DISCUSSION}

Figure 5 illustrates the inter-observer agreement of the manual annotations of VPPG records and the agreement between both methods and observers. 

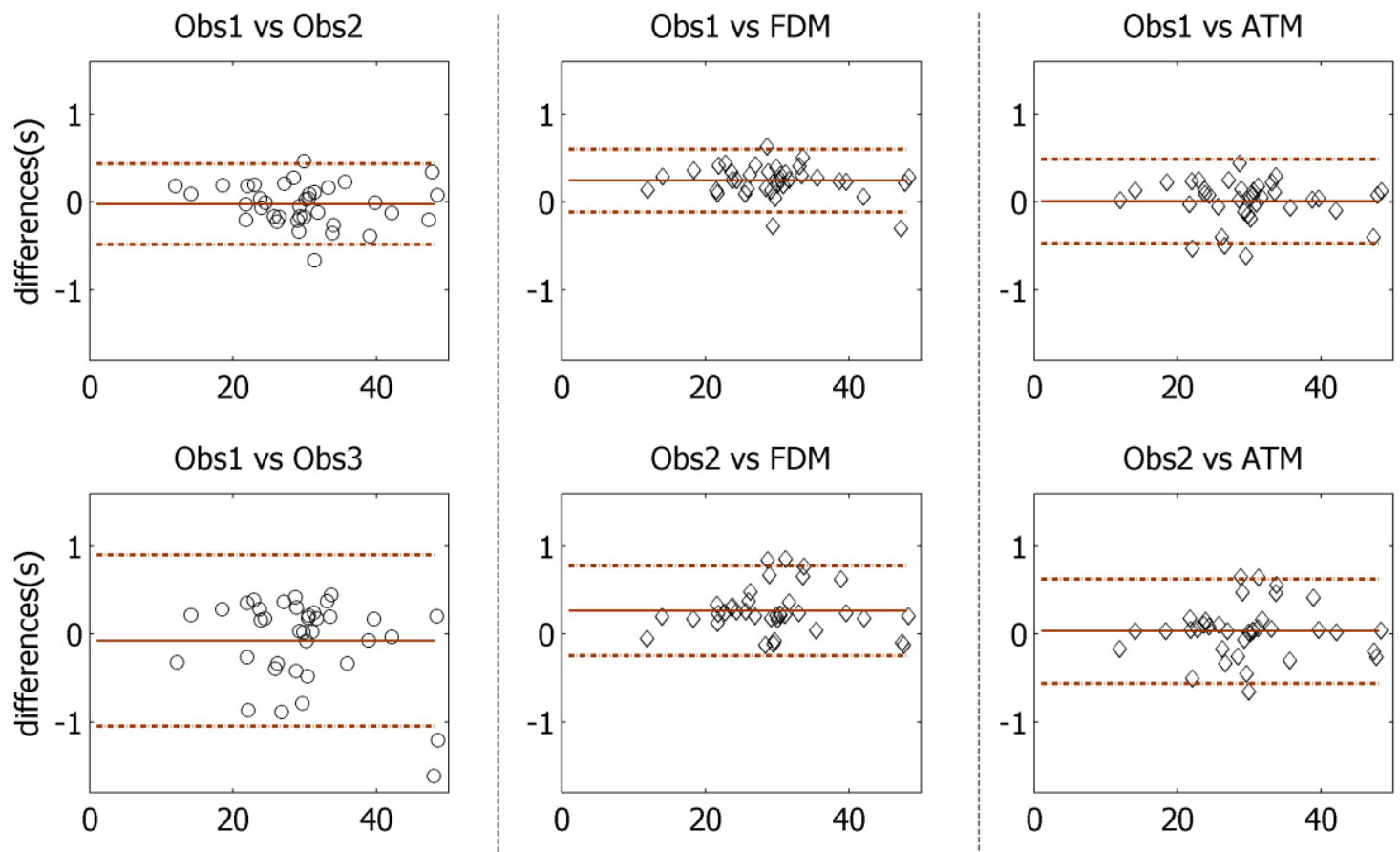

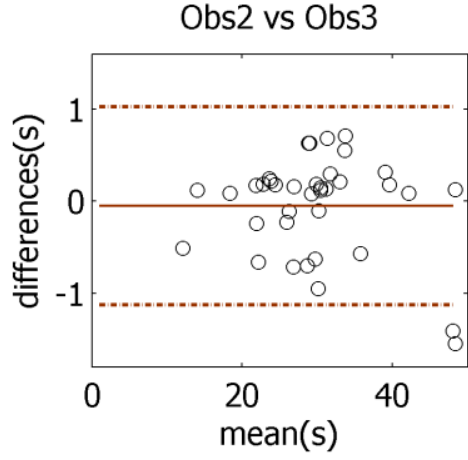

(a)

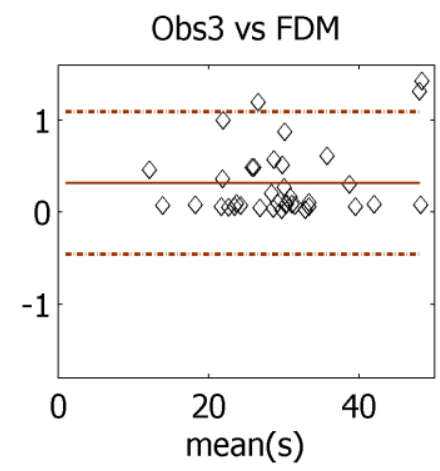

(b)

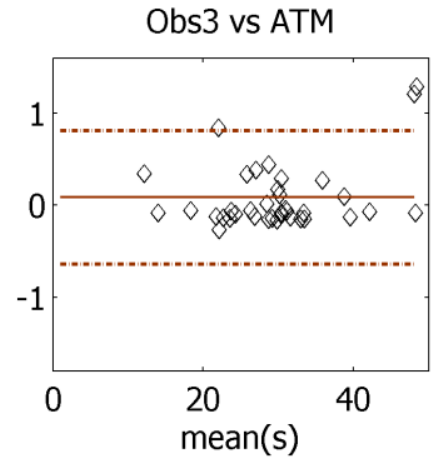

(c)

Figure 5. Bland-Altman graphics for assessment of: (a) inter-observer agreement for the manual annotation of the EEM, (b) agreement between FDM and observers and, (c) agreement between ATM and observers.

Table 1 contains inter-observer errors and inter-observer LoA computed as in [19]. Table 2 shows errors from methods with respect to observers and LoA. The error (e) was estimated as a function of the means $(\bar{x})$ and standard deviations (s) of the resultant differences in every case as in (3).

$$
e=\bar{x} \pm 2 * s,
$$

From the analysis of the data in Table 1 and Figure 5 it was noted that:

- Differences between manual annotations from observer 1 and observer 2 on a same signal are within the range of -0.49 to 0.44 seconds, with $95 \%$ probability (normal distribution of data assumed).

- The results of observer 3 disagree the most with the others two observers. A confidence interval of $95 \%$ of the differences in these cases (LoA) was approximately 1 s. As can be seen in Table I and Figure 5(a), observer 3's results presented the largest deviation from the mean of differences. 
Nevertheless, observer 3 was kept as reference to assess the methods. As a precaution, his criterion was assigned the smallest significance among all the observers.

It is important to note that the detection of the EEM is an essential step in VRT computation. This hemodynamic parameter is in the order of tens, two orders of magnitude above the difference between observers 1 and 2 . For that reason it can be concluded that the agreement between them is good. The agreement between observer 3 and the others can be considered acceptable.

From the analysis of data shown in Table 2, Figure 5(b) and Figure 5(c) it was noted that:

- The agreement among both methods and observers are consider acceptable. The confidence interval of $95 \%$ of errors in all cases was approximately $1 \mathrm{~s}$.

- In every comparison, FDM results gave the largest mean error. Nevertheless, the standard deviation was similar for both methods.

Table 1. Inter-observer errors and LoA.

\begin{tabular}{ccc}
\hline Comparisons & Errors $(\mathrm{s})$ & LoA $(\mathrm{s})$ \\
\hline Obs1 vs Obs2 & $-0.02 \pm 2 * 0.23$ & $(-0.49 ; 0.44)$ \\
Obs1 vs Obs3 & $-0.08 \pm 2 * 0.49$ & $(-1.05 ; 0.90)$ \\
Obs2 vs Obs3 & $-0.05 \pm 2 * 0.54$ & $(-1.13 ; 1.03)$ \\
\hline
\end{tabular}

Table 2. Errors and LoA from methods respect to observers.

\begin{tabular}{cccc}
\hline & Comparisons & Errors (s) & LoA $(\mathrm{s})$ \\
\hline \multirow{2}{*}{1} & FDM vs Obs1 & $0.24 \pm 2 * 0.18$ & $(-0.12 ; 0.60)$ \\
& ATM vs Obs1 & $0.01 \pm 2 * 0.24$ & $(-0.47 ; 0.49)$ \\
\hline \multirow{2}{*}{2} & FDM vs Obs2 & $0.27 \pm 2 * 0.26$ & $(-0.25 ; 0.78)$ \\
& ATM vs Obs2 & $0.03 \pm 2 * 0.30$ & $(-0.56 ; 0.63)$ \\
\hline \multirow{2}{*}{3} & FDM vs Obs3 & $0.32 \pm 2 * 0.39$ & $(-0.45 ; 1.09)$ \\
& ATM vs Obs3 & $0.08 \pm 2 * 0.36$ & $(-0.64 ; 0.81)$ \\
\hline
\end{tabular}

Aiming to increase the statistical consistency of results presented above, Table 3 and Table 4 show the p-values resulting from testing the statistical significance of differences between observer annotations and methods' errors. Table III show the p-values resulting from two-way ANOVA by setting factor 1 as observers and factor 2 as method.

Table 3. Two-way anova

\begin{tabular}{lcc}
\hline & Observers & Methods \\
\hline p-value & $2.8^{*} 10^{-1}$ & $1.6^{*} 10^{-8}$ \\
\hline
\end{tabular}

From Table 3 can be stated that there is no significant differences between observers annotations ( $p$-value $=0.28$ ), but in contrast (as expected) there is significant differences between methods' errors ( $p$-value $<<0.01$ ).

Table 4 strengthens the fact of no differences related to observers, and supports the existence of significant statistical differences between FDM and ATM. The differences between methods are not related to which observer annotation is taken as reference to compute the methods' error (as can be seen in light blue columns in Table 4). 
Table 4. P-values from One-way ANOVA (parametric) and Kruskal-Wallis (non-parametric) test from comparisons between observers. Multiple comparison test (highlighted columns), for differences between methods' error.

\begin{tabular}{cccccccc}
\hline & Inter & $E_{F D M 1}$ & $E_{A T M 1}$ & $E_{F D M 1}$ & $E_{F D M 1}$ & $E_{F D M 2}$ & $E_{F D M 2}$ \\
& Observer & $E_{F D M 2}$ & $E_{A T M 2}$ & $E_{A T M 1}$ & $E_{A T M 2}$ & $E_{A T M 1}$ & $E_{A T M 2}$ \\
\hline Parametric & $8.8^{*} 10^{-1}$ & $9.7^{*} 10^{-1}$ & $9.7^{*} 10^{-1}$ & $2.9^{*} 10^{-4}$ & $1.6^{*} 10^{-3}$ & $4.4^{*} 10^{-5}$ & $2.9^{*} 10^{-4}$ \\
Non-Parametric & $6.4^{*} 10^{-1}$ & $9.6^{*} 10^{-1}$ & $1.0^{*} 10^{-0}$ & $3.4^{*} 10^{-5}$ & $5.9^{*} 10^{-5}$ & $3.1^{*} 10^{-4}$ & $5.1^{*} 10^{-4}$ \\
\hline
\end{tabular}

Based on the manual annotations used as a reference, Bland-Altman graphics and the performed statistical tests, it can be argued that ATM is more accurate than FDM for automatic detection of the EEM. This statement agrees with the results evidenced in [17], where ATM showed better results than a derivative based method for onset detection of arterial pulse waves. Derivative based methods are vulnerable to noise and artifacts commonly present in biomedical signals. The robustness of ATM is based in the fact that this method realizes computations supported in morphological trends of biomedical signals, rather than the difference between successive samples as in FDM.

\section{CONCLUSION}

Manual analysis of biomedical signal is common practice both in clinical and research studies. Manual annotations on physiological waveforms are time-consuming, and when this method is used care needs to be taken to avoid subject bias and other shortcomings related to by hand analysis. When possible, automatic analysis should substitute manual scrutiny of physiological signals. Here were presented tow methods (ATM and FDM) for automatic analysis of venous photoplethismografic waveforms. Both methods show a performance comparable to human observers for the detection of the End of the Emptying Maneuvers. Nevertheless, ATM proved to be a more exact method due to its computational robustness, and demonstrated good results for biomedical signal analysis. Particularly ATM method seems to be an effective option to replace manual point detection in venous photoplethysmographic signals.

Funding: This research received no external funding.

Conflicts of Interest: The authors declare no conflict of interest.

\section{REFERENCES}

1. J. L. Beebe-Dimmer, J. R. Pfeifer, J. S. Engle, and D. Schottenfeld, "The Epidemiology of Chronic Venous Insufficiency and Varicose Veins," Ann. Epidemiol., vol. 15, no. 3, pp. 175184, Mar. 2005.

2. B. Santler and T. Goerge, "Chronic venous insufficiency - a review of pathophysiology, diagnosis, and treatment," JDDG J. der Dtsch. Dermatologischen Gesellschaft, vol. 15, no. 5, pp. 538-556, May 2017.

3. A. M. Prabhakar et al., "Changing Medicare Utilization of Minimally Invasive Procedures for the Treatment of Chronic Venous Insufficiency," J. Vasc. Interv. Radiol., vol. 28, no. 6, pp. 818-824, Jun. 2017.

4. C. R. Lattimer, C. Franceschi, and E. Kalodiki, "Optimizing calf muscle pump function," Phlebology, vol. 0, no. 0, p. 026835551770941 , May 2017. 
5. R. T. Eberhardt and J. D. Raffetto, "Chronic Venous Insufficiency," Circulation, vol. 130, no. 4, pp. 333-346, Jul. 2014.

6. K. A. L. Darvall, R. C. Sam, G. R. Bate, D. J. Adam, S. H. Silverman, and A. W. Bradbury, "Photoplethysmographic venous refilling times following ultrasound guided foam sclerotherapy for symptomatic superficial venous reflux: Relationship with clinical outcomes," Eur. J. Vasc. Endovasc. Surg., vol. 40, no. 2, pp. 267-272, 2010.

7. H. AB and S. C, "Observations on the finger volume pulse recorded photoelectrically," $A m J$ Physiol, vol. 119, pp. 334-335, 1937.

8. J. Allen, "Photoplethysmography and its application in clinical physiological measurement," Physiol. Meas., vol. 28, no. 3, pp. 1-39, 2007.

9. U. Schultz-Ehrenburg and V. Blazek, "Value of quantitative photoplethysmography for functional vascular diagnostics: Current status and prospects," Skin Pharmacol. Appl. Skin Physiol., vol. 14, no. 5, pp. 316-323, 2001.

10. A. A. Kamshilin and N. B. Margaryants, "Origin of Photoplethysmographic Waveform at Green Light," Phys. Procedia, vol. 86, pp. 72-80, 2017.

11. A. A. Alian and K. H. Shelley, "Photoplethysmography," Best Pract. Res. Clin. Anaesthesiol., vol. 28, no. 4, pp. 395-406, 2014.

12. C. Cetin, M. O. Serbest, S. Ercan, T. Yavuz, and A. Erdogan, "An evaluation of the lower extremity muscle strength of patients with chronic venous insufficiency," Phlebol. J. Venous Dis., vol. 31, no. 3, pp. 203-208, Apr. 2016.

13. O. Adas, S. Jr, M. G. Ii, H. Almeida, and R. lii, "Noninvasive diagnostic methods to evaluate venous insufficiency of the lower limbs," J Vasc Bras, vol. 6, no. 3, pp. 26-275, 2007.

14. S. Ercan, C. Çetin, T. Yavuz, H. M. Demir, and Y. B. Atalay, "Effects of isokinetic calf muscle exercise program on muscle strength and venous function in patients with chronic venous insufficiency," Phlebol. J. Venous Dis., vol. 33, no. 4, pp. 261-266, May 2018.

15. M. Elgendi, "On the Analysis of Fingertip Photoplethysmogram Signals," Curr. Cardiol. Rev., vol. 8, no. 1, pp. 14-25, 2012.

16. I. Sanchez Gendriz, D. Hernandez Seoane, H. F. Posada Quintero, and A. Sonora Mengana, "Automatic detection of the end of the Empting Maneuvers in venous muscle pump test," in 2012 XVII Symposium of Image, Signal Processing, and Artificial Vision (STSIVA), 2012, pp. 30-33.

17. D. D. Rodríguez, M. B. C. Sanz, C. R. V. Seisdedos, M. S. Perrone, and L. N. Siri, "Automatic Recognition of the Onset Point in the Arterial Pulse Wave," in IFMBE Proceedings, vol. 33, 2013, pp. 1130-1133.

18. J. H. McClellan and T. W. Parks, "A personal history of the Parks-McClellan algorithm," IEEE Signal Process. Mag., vol. 22, no. 2, pp. 82-86, Mar. 2005.

19. J. M. Bland and D. G. Altman, "Statistical methods for assessing agreement between two methods of clinical measurement," Int. J. Nurs. Stud., vol. 47, no. 8, pp. 931-936, 2010.

20. E. Vittinghoff, D. V. Glidden, S. C. Shiboski, and C. E. McCulloch, Regression methods in biostatistics: linear, logistic, survival, and repeated measures models., Second edi. Springer Science \& Business Media, 2011.

21. W. W. Daniel and C. L. CROSS, Biostatistics: A Foundation for Analysis in the Health Sciences, Tenth Edit. John Wiley \& Sons, 2013. 
*Correspondence: ignaciogendriz@gmail.com 